E6-2002-75

V. A. Karnaukhov ${ }^{1}$, S. P. Avdeyev, E. V. Duginova, L. A. Petrov, V. K. Rodionov, H. Oeschler ${ }^{2}$, A. Budzanowski ${ }^{3}$, W. Karcz ${ }^{3}$, M. Janicki ${ }^{3}$, O. V. Bochkarev ${ }^{4}$, E. A. Kuzmin ${ }^{4}$, L. V. Chulkov ${ }^{4}$, E. Norbeck ${ }^{5}$, A. S. Botvina ${ }^{6}$

\title{
THERMAL MULTIFRAGMENTATION OF HOT NÚCLEI AND LIQUID-FOG PHASE TRANSITION
}

Submitted to «Ядерная физика»

\footnotetext{
${ }^{1}$ E-mail: karna@nusun.jinr.ru

${ }^{2}$ Institut für Kernphysik, TU Darmstadt, 64289, Germany;

E-mail: h.oeschler@gsi.de

${ }^{3} \mathrm{H}$. Niewodniczanski Institute of Nuclear Physics, 31-342, Cracow, Poland

${ }^{4}$ Kurchatov Institute, 123182, Moscow, Russia

${ }^{5}$ University of Iowa, Iowa City, IA 52242, USA

${ }^{6}$ GSI, Postfach 110552, D-64220 Darmstadt, Germany
} 


\section{Introduction}

Study of the decay properties of the hot nuclei is one of the most challenging topics of modern nuclear physics. The excitation energy of the hot nuclei $(500-700 \mathrm{MeV})$ is comparable with the total binding energy. They disintegrate via a new multibody decay mode - thermal multifragmentation. This process is characterized by the copious emission of intermediate mass fragments which are heavier than alpha particles but lighter than fission fragments (IMF, $2<\mathrm{Z} \leq 20$ ). Such multibody disintegration is not an exotic but the main decay channel of a very hot nuclear system.

The development of this field has been strongly stimulated by an idea that this process is related to the nuclear liquid-gas phase transition. One of the first nuclear models, suggested by N. Bohr, K. Weizsaecker, and Ya.I. Frenkel 65 years ago, is the liquid-drop model, which has been successfully used up to now. The liquid - gas phase transition in nuclear matter was predicted much later [1-3] on the basis of the similarity between van der Waals and nucleonnucleon interactions. In both cases the attraction between particles is replaced by repulsion at a small interaction range. As a result, the equations of state are similar for so different systems. It is well seen in the phase diagram (Fig.1) taken from [2]. The figure shows the isotherms for pressure as a function of volume calculated for the van der Waals system and the Fermi gas of nucleons interacting through Skyrme forces. The scales are the same for both cases due to the use of dimensionless variables: pressure, volume and temperature are given as ratios to the critical values $P_{c}, V_{c}=1 / \rho_{c}$ ( $\rho_{c}$ is the critical density) and $T_{c}$. The very steep part of the isotherms (on the left) corresponds to the liquid phase. The gas phase is presented by the right parts of the isotherms where pressure is changing smoothly with increasing volume. A point of peculiar interest is the part of the diagram below the hatched line where the isotherms correspond to negative compressibility. The density here is significantly reduced as compared to the liquid phase. This is a spinodal region characterized by the phase instability. One can imagine that a hot nucleus expands due to thermal pressure and enters into the metastable region. Due to density fluctuations, a homogeneous system converts into the mixed-phase state, consisting of droplets (IMF's) surrounded by nuclear gas (nucleons and light composite particles). In fact, the final state of this transition is a nuclear fog [3]. 


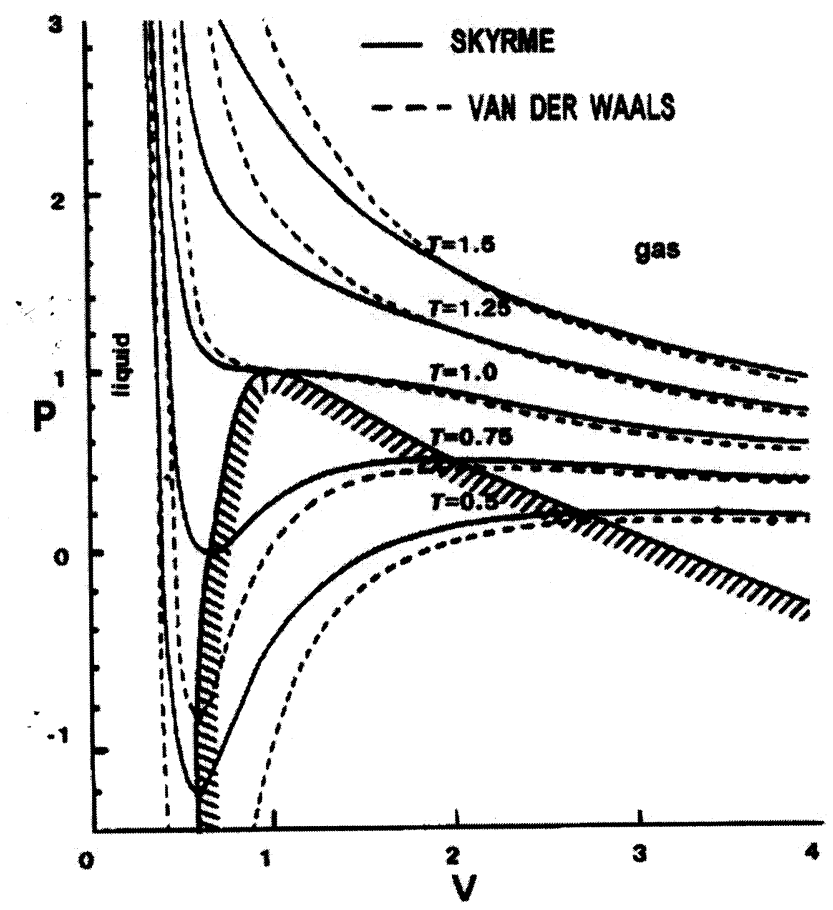

Fig.1. Comparison of the equation of state for a van der Waals gas and for a nuclear system interacting through a Skyrme force (the relative units are used). 
The neutrons fly away with the energies corresponding to the system temperature (5-6 $\mathrm{MeV}$ ), while the charged particles are additionally accelerated in the Coulomb field of the system. Disintegration time is determined by the time scale of the thermodynamic fluctuations and is expected to be very short. This is a scenario of nuclear multifragmentation as the spinodal decomposition, considered in a number of theoretical and experimental papers (see, for example, [4-10] and review papers [11,12]). This picture was proved experimentally with a significant contribution from the FASA collaboration; the short review of the results obtained is presented below.

As for the critical temperature for the liquid-gas phase transition $T_{c}$ (at which the surface tension vanishes), its value is not definitely known. There are many theoretical calculations of $T_{c}$ for finite nuclei. In Ref. [1,2] it is done using a Skyrme effective interaction and thermal Hartree-Fock theory. The values of $T_{c}$ were found to be $18.1 \mathrm{MeV}$ [1] and in the range (8.1-20.5) MeV [2] depending on the chosen Skyrme interaction parameters. There are not yet reliable experimental data for $T_{c}$, though it is claimed in a number of papers. The latest of them is Ref. [13], where it is stated that $T_{c}=6.7 \pm 0.2 \mathrm{MeV}$. We return to the discussion of this point in Chapter 6.

\section{How to produce and study hot nuclei}

An effective way to produce hot nuclei is collisions of heavy ions with energies up to hundreds of $\mathrm{MeV}$ per nucleon. Around a dozen of sophisticated experimental devices were created to study nuclear multifragmentation with heavy ion beams. But in this case heating of nuclei is accompanied by compression, strong rotation and shape distortion, which may essentially influence the decay properties of hot nuclei.

Investigation of dynamic effects caused by excitation of collective (or "mechanical") degrees of freedom is interesting in itself, but there is a great problem of disentangling all these effects to get information on the thermodynamic properties of a hot nuclear system. One gains simplicity, and the picture becomes clearer, when light relativistic projectiles (first of all protons, antiprotons, pions) are used. In contrast to heavy ion collisions, fragments are emitted by the only source - the slowly moving target spectator. Its excitation energy is almost entirely thermal. Light relativistic projectiles provide therefore a unique 
possibility of investigating "thermal multifragmentation", which was realized in the FASA project.

Figure 2 illustrates the central collision of a relativistic proton with a heavy target (usually it is $\mathrm{Au}$ ). Passing through the target, the proton creates an intranuclear cascade. Fast cascade particles are ejected into the forward hemisphere, but slower ones are absorbed by the target spectator. As a result, the hot remnant nucleus expands and disintegrates emitting nucleons and fragments.

To study multifragmentation with the beams of the Dubna synchrophasotron, a $4 \pi$ setup FASA was created [14]. The device consists of two main parts:

1) Five $d E-E$ telescopes (at $\theta=24^{\circ}, 68^{\circ}, 87^{\circ}, 112^{\circ}$ and $156^{\circ}$ with the beam direction), which serve as triggers for the read-out of the system allowing the measurement of the fragment charge and energy distributions. Ionization chambers and $\mathrm{Si}(\mathrm{Au})$ detectors are used as $d E$ and $E$ counters respectively.

2) A fragment 'multiplicity detector (FMD) including $64 \mathrm{CsI}(\mathrm{Tl})$ counters (with a scintillator thickness averaging $35 \mathrm{mg} \cdot \mathrm{cm}^{-2}$ ), which cover $89 \%$ of $4 \pi$. The FMD gives the number of IMF's in the event and their angular distribution.

A self-supporting Au target $1.0 \mathrm{mg} / \mathrm{cm}^{2}$ thick is located at the center of the FASA vacuum chamber ( $1 \mathrm{~m}$ in diameter).

The following beams were used: protons at energies 2.16, 3.6 and $8.1 \mathrm{GeV}[15],{ }^{4} \mathrm{He}$ at energies 4 and $14.6 \mathrm{GeV}$ and ${ }^{12} \mathrm{C}$ at $22.4 \mathrm{GeV}[16]$. The mean beam intensity was around $7 \cdot 10^{8} \mathrm{p} /$ spill for protons and helium and $1 \cdot 10^{8} \mathrm{p} /$ spill for carbon projectiles (spill length 300 $\mathrm{ms}$, spill period $10 \mathrm{~s}$ ).

In the last years FASA has been significantly upgraded, a new counter array consisting of $25 \mathrm{dE}-\mathrm{E}$ telescopes was developed. At present, the total number of detectors in FASA is 129 which are supplied with 193 electronic channels.

\section{Evidence for thermalization of the target spectator at break-up}

Let us consider a very important point of thermalization of the system at break-up. To check whether this state is close to the thermal equilibrium, the plots were composed for the fragment yields in terms of the longitudinal-versus-transverse velocity components. They look similar for all collisions investigated. Figure 3 shows such plots for ${ }^{4} \mathrm{He}+\mathrm{Au}$ and ${ }^{12} \mathrm{C}+$ 

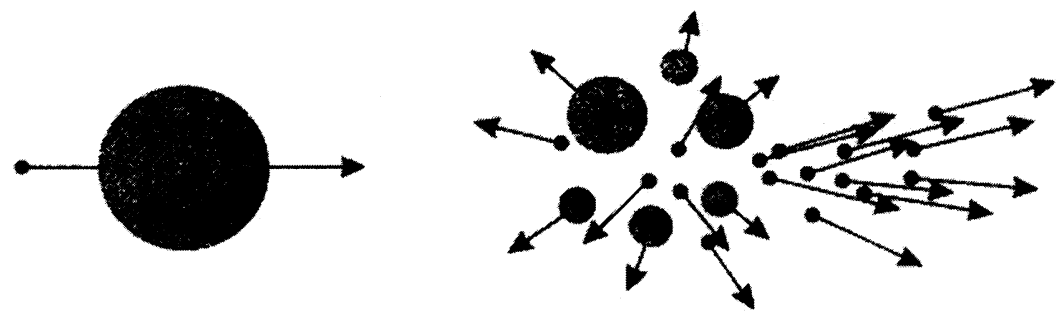

Fig. 2. Illustrative picture of a central collision of a relativistic proton with a heavy target.
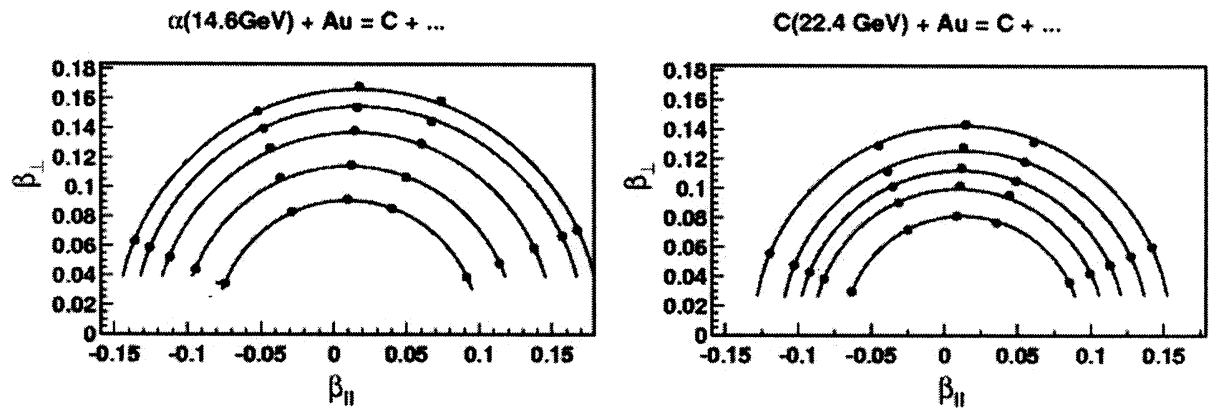

Fig. 3. Transverse versus longitudinal velocity plot for carbon isotopes produced in ${ }^{4} \mathrm{He}(14.6 \mathrm{GeV})$ and ${ }^{12} \mathrm{C}(22.4 \mathrm{GeV})$ collisions with $\mathrm{Au}$ target. Circles are drawn through points of equal invariant cross section corresponding to isotropic emission in the moving source frame. 


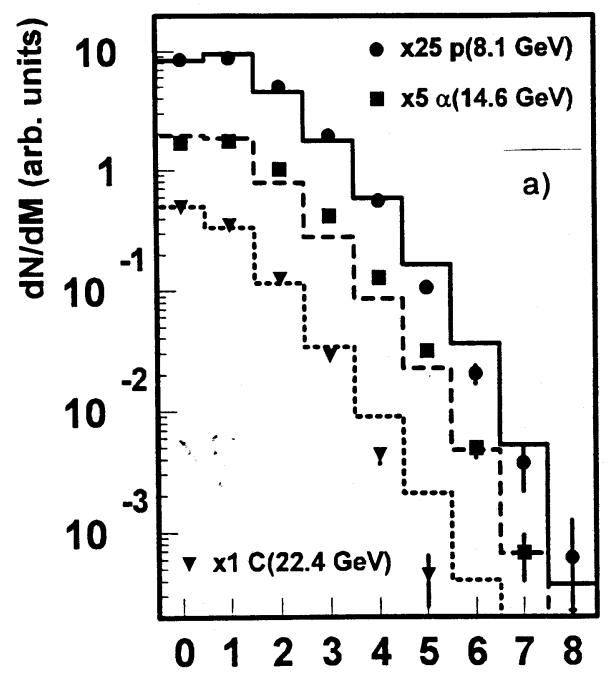

FMD-multiplicity (IMF)

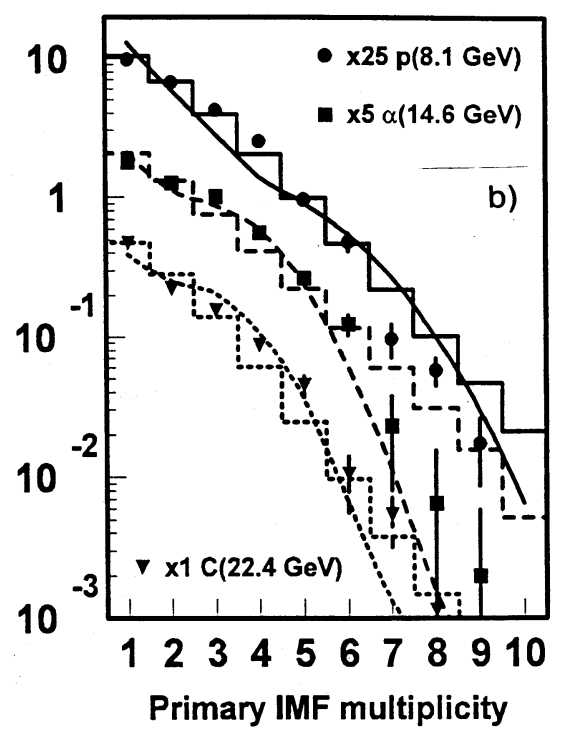

Fig.4. a) Measured IMF-multiplicity distributions and fits with a Fermi functions (folded with the experimental filter) associated with a trigger fragment for $\mathrm{p}+\mathrm{Au}$ collisions at $8.1 \mathrm{GeV},{ }^{4} \mathrm{He}+\mathrm{Au}$ at $14.6 \mathrm{GeV}$, and ${ }^{12} \mathrm{C}+\mathrm{Au}$ at $22.4 \mathrm{GeV}$. b) Symbols represent directly reconstructed primary IMF distributions; histograms are the Fermi distributions used to fit the data in the left part. The lines are calculated with the INC + Expansion + SMM model. 
$\mathrm{Au}$ interactions [16]. The symbols correspond to the constant invariant cross sections taken for emitted carbon fragments. The lines connecting the experimental points form circles demonstrating isotropic emission in the frame of the moving source. This indicates that the fragment emission proceeds from a thermalized state. The center positions of circles determine the source velocities, which are found to be in the range of (0.01-0.02) c. The IMF angular distribution in the laboratory system exhibits a forward peak caused by the source motion.

Another finding in favor of the thermal equilibrium is that the fragment kinetic energy spectra look like maxwellian ones with maximum around the Coulomb barrier followed by an exponential tail. All these observations can be considered as good motivation for using statistical approaches to describe the data. It was done in our studies rather successfully. Some details of the model used are given in the next section, but here for the illustration we present in Fig. 4 the fragment multiplicity distributions for different collisions in comparison with the model calculations.

\section{Density of the system at break-up}

What is the size of a fragmenting target spectator? Is it true that a very hot nucleus expands due to the thermal pressure to get into the phase instability (spinodal) region? To answer this question we measured [17] the distribution of the relative velocities for coincident fragments at large correlation angles. The fragment kinetic energy is determined in the main by the acceleration in the Coulomb field of the fragmenting nucleus. Therefore, the fragment velocity is sensitive to the configuration of the system at the break-up moment. In the upper part of Fig. 5 two variants of fragment emission are shown: evaporation from the surface of the nucleus with normal density (right) and the volume decay of the expanded system (left).

The measured distribution is shifted to the lower velocities relative to the calculated one for the surface emission, this observation is in favor of the volume fragment emission. After quantitative analysis of the data by means of combined model (see below) it was concluded that the fragment emission occurs from the expanded system with the mean density 3-4 times smaller than the normal one [18,19]. The same conclusion is drawn from the considerations of the fragment kinetic energy spectra [15]. So, one can say that thermal 


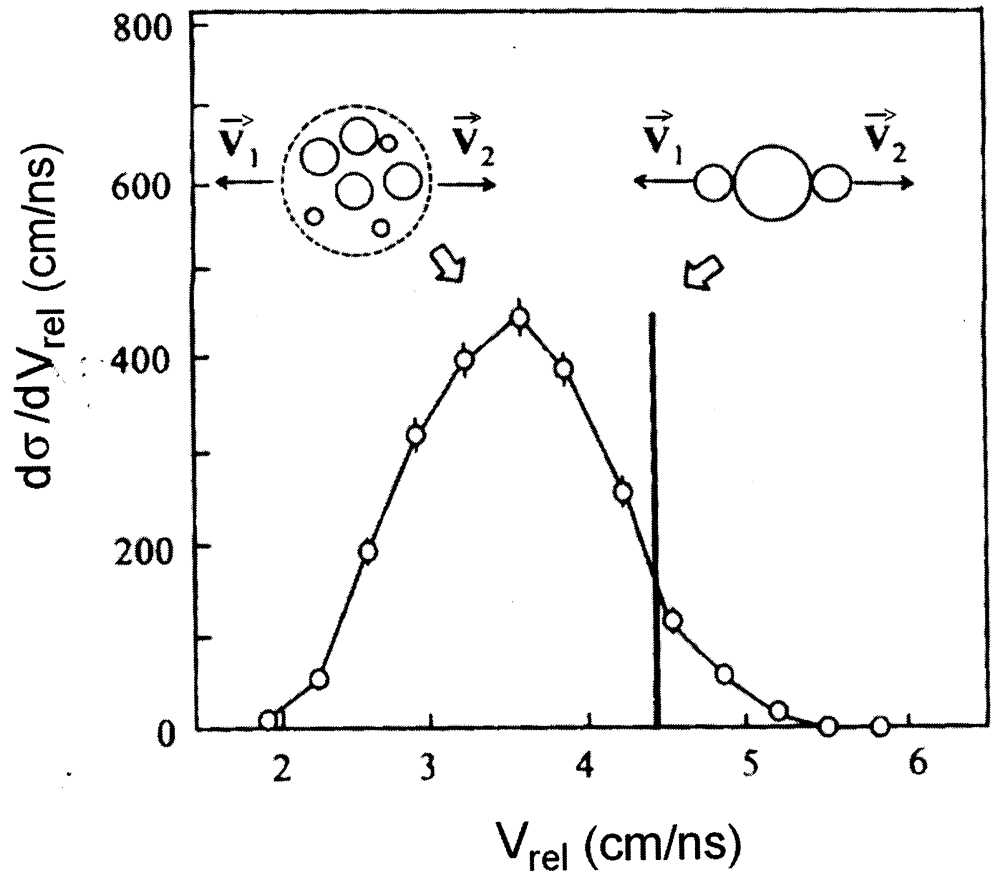

Fig.5. Distribution of relative velocities for the coincident fragments from ${ }^{4} \mathrm{He}+\mathrm{Au}$ collisions (at $14.6 \mathrm{GeV}$ ) measured at correlation angles $150^{\circ}-180^{\circ}$. The vertical line shows the expected maximum position for fragment evaporation from the nucleus surface. The data are shifted to lower velocities, corresponding to the volume disintegration of the expanded system. 
multifragmentation is indeed the spinodal decomposition process. This conclusion is supported by measuring the time scale of fragment emission, which is very fast (see the next chapter).

Now, let us consider the combined model. The reaction mechanism for light relativistic projectiles is usually divided into two steps. The first one is a fast energy deposition stage, during which energetic light particles are emitted and the nuclear remnant is excited. The fast stage is usually described by the intranuclear cascade model (INC). We use the version of the INC from [20] to get the distribution of the nuclear remnants in charge, mass and excitation energy. The second stage is described by the statistical multifragmentation model (SMM) [21]. Within the SMM the probability of different decay channels of the excited remnant is proportional to their statistical weight. The break-up volume determining the Coulomb energy of the system is taken to be $V_{b}=(1+k) A / \rho_{o}$, where $A$ is the mass number of the decaying nucleus, $\rho_{o}$ is the normal nuclear density, $k$ is the model parameter. So, thermal expansion before the break-up is assumed. The break-up density is $\rho_{b}=\rho_{o} /(1+k)$. It is found that this traditional approach fails to describe the observed IMF multiplicities, whose mean values saturate at $(2.2 \pm 0.2)$. The expansion stage is inserted between the two parts of calculation. In fact, the excitation energies and the residual masses are finely tuned to get agreement with the measured IMF multiplicities, i.e., the values for the residual (after INC) masses and their excitation energies are scaled on an event-by-event basis (see for details $[15,16])$. The final stage of the combined model INC + Expansion + SMM is the multibody Coulomb trajectory calculations for all charged particles in the exit channel (again on an event-by-event basis). As a result, the fragment energies and momenta are obtained and can be compared with the experimental data.

\section{Thermal multifragmentation-a new decay mode of hot nuclei}

The time scale of IMF emission is a crucial characteristic for understanding the mechanism of this decay process: whether it is a "slow" successive and independent evaporation of IMF's or a new (multibody) decay mode with "simultaneous" ejection of the fragments governed by the total accessible phase space. "Simultaneous" means that all the fragments are liberated during the time which is smaller than the characteristic Coulomb time $\tau_{c} \approx 10^{-21} \mathrm{~s}[22]$, which is the mean time of fragment acceleration in the Coulomb field 
of the system. In that case, emission of the fragments is not independent, they interact with each other via the Coulomb forces during the acceleration. Thus, measurement of the IMF emission time $\tau_{\mathrm{em}}$ (the mean time separation between two consecutive fragment emissions) is a direct way to answer the question as to the nature of the multifragmentation phenomenon.

There are two procedures to measure the emission time: analysis of the IMF-IMF correlation function in respect to the relative velocity and the relative angle. We used the second method. Figure 6 shows the IMF-IMF relative angle correlation for the fragmentation target spectator in $\mathrm{He}(14.6 \mathrm{GeV})+\mathrm{Au}$ collisions [18]. The correlation function exhibits a minimum at $\theta_{\text {rel }}=0$ arising from the Coulomb repulsion between the coincident fragments. The magnitude of this effect drastically depends on the time scale of emission, since the longer the time distance between the fragments, the larger their space separation and the weaker the Coulomb repulsion. The multibody Coulomb trajectory calculations fit the data on the assumption that the mean emission time is less than $75 \mathrm{fm} / c$ $\left(2.3 \cdot 10^{-22} \mathrm{~s}\right)$. This value is significantly smaller than the characteristic Coulomb time $\tau_{c}$. The trivial mechanism of IMF emission (independent evaporation) is definitely excluded.

A similar result is obtained in our recent paper [23], devoted to the time scale measurement for multifragment emission in $p+\mathrm{Au}$ collisions at $8.1 \mathrm{GeV}$. The model dependence of the results was carefully investigated. Figure 7 shows the experimental correlation function and the calculated ones for two values of the break-up volumes: $V_{b}=4 V_{o}$ and $V_{b}=8 V_{o}$. The mean emission time is found to be $\tau_{\mathrm{em}}=(50 \pm 10) \mathrm{fm} / c$. One should notice that this value is in fact the mean time of fragment formation at break-up conditions. The total duration time of the reaction is larger. It includes the thermalization time $(10-20 \mathrm{fm} / c)$ and the mean expansion time before the disintegration of hot nucleus, which is around 75 $\mathrm{fm} / \mathrm{c}$ according to model estimation $[15,24]$.

\section{Evolution of the reaction mechanism with increasing projectile mass}

It is shown in a number of papers, that the multifragment emission in the central collisions of very heavy ions is not described by the statistical models. Initial compression of the system is tremendous and the collective part of the excitation energy is so large that the partition of the system into fragments is likely to be a very fast dynamic process [25]. In that 


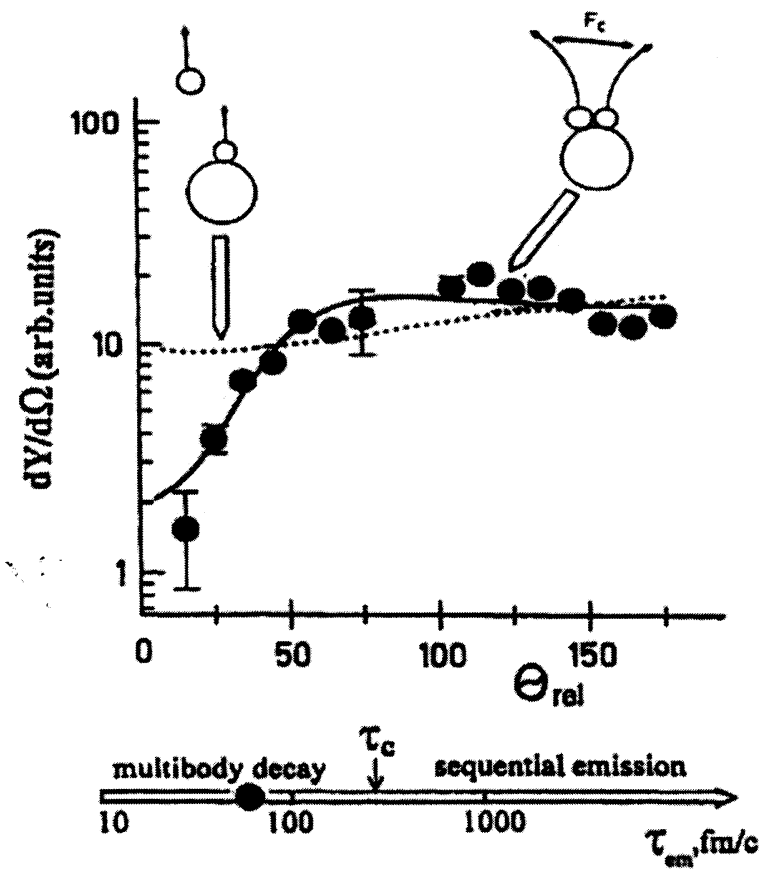

Fig. 6. Distribution of relative angles between coincident IMF's for ${ }^{4} \mathrm{He}(14.6 \mathrm{GeV})+\mathrm{Au}$ collisions. Solid line is calculated for the simultaneous emission of fragments, dashed line corresponds to the sequential, independent evaporation. 


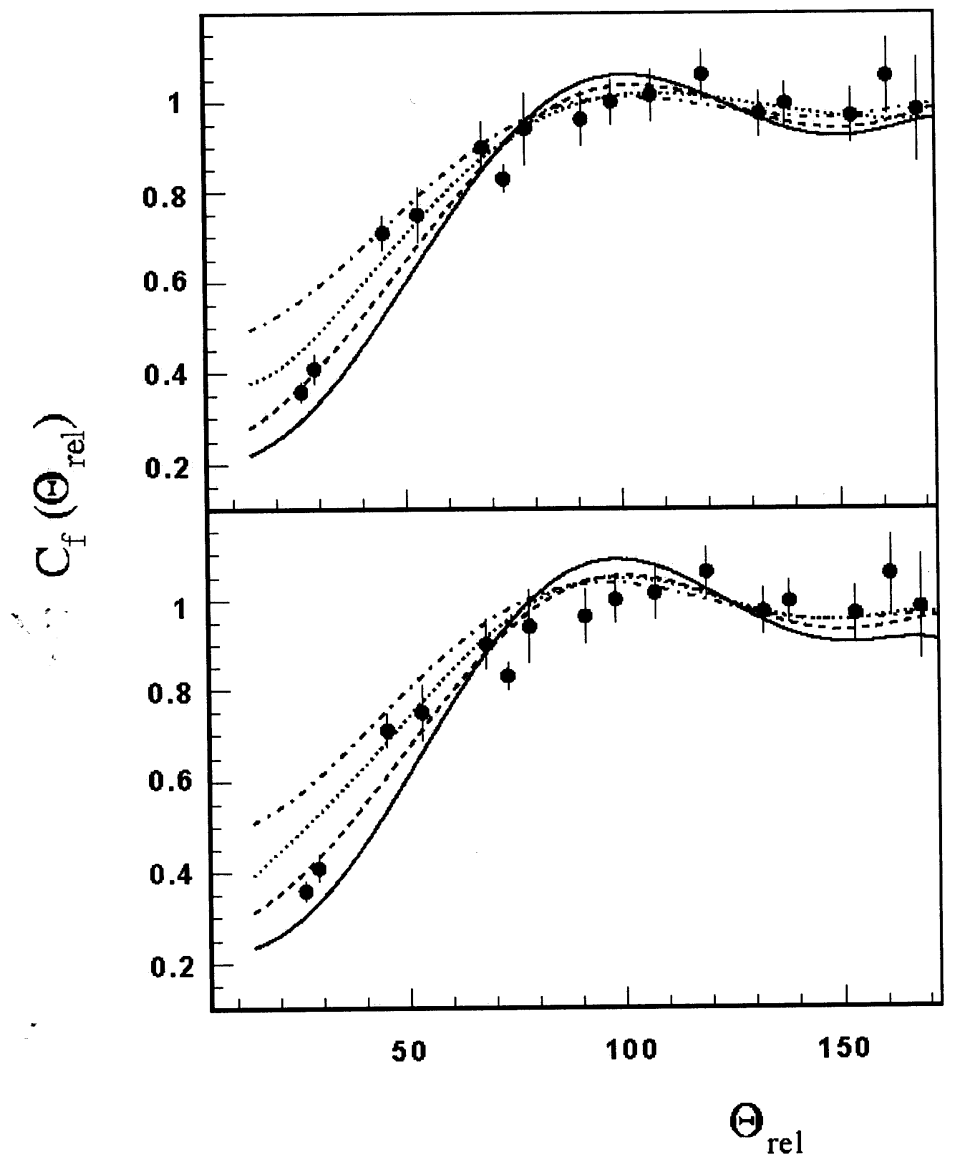

Fig. 7. Comparison of the measured correlation functions (for $p+\mathrm{Au}$ at $8.1 \mathrm{GeV}$ ) with the calculated ones for different mean decay times of the fragmenting system: solid, dashed, dotted and dash dotted lines for $\tau_{e m}=0,50,100$ and $200 \mathrm{fm} / \mathrm{c}$. Calculations are made with INC+Exp.+SMM model assuming two values of break-up volumes: $8 \mathrm{~V}_{0}$ (upper panel) and $4 \mathrm{~V}_{0}$ (lower panel). 
case the fragment kinetic energy is largely determined by the decompressional collective flow. It is interesting to follow the evolution of the multifragmentation mechanism (as the projectile mass increases) from pure thermal to that influenced by the dynamic effects.

\subsection{Fragment charge distribution}

We performed the comparative study of multifragmentation induced in a gold target by relativistic protons, helium and carbon ions [16,26]. It was already demonstrated that in all the cases one dealt with disintegration of a thermally equilibrated system (Fig.3), and IMF multiplicity distributions were well reproduced by the statistical model (Fig.4). It was also found that charge distributions of fragments were similar for all the collisions studied, and they are very well described by the combined INC + expansion + SMM model ( Fig.8).

The general trend of the IMF charge distributions is also well reproduced by a power law $Y(Z) \sim Z^{-\tau}$. In earlier papers on multifragmentation [3,27-29] such power-law dependence for the fragment charge yield was interpreted as an indication of the proximity of the decaying state to the critical point for the liquid-gas phase transition in nuclear matter. This was stimulated by the application of the classical Fisher drop model [30], which predicted a pure power law droplet-size distribution with $\tau=2-3$ at the critical point. According to this model, the $\tau$-parameter has a minimal value at the critical temperature. So, in the spirit of the Fisher model, the data in the insert of Fig. 8 should be considered as an indication of the "critical behavior" of the system at the beam energies (5-10) GeV. But it is not the case. The power law is well explained at temperatures far below the critical point. As is seen in Fig.8, the pure thermodynamical SMM predicts that the IMF charge distribution is close to a power law at freeze-out temperatures of 5-6 MeV, while the critical temperature is assumed to be $T_{c}=18 \mathrm{MeV}$. The statistical model also predicts the parabolic dependence of the exponent $\tau$ on the excitation energy with the minimal value around 2.0 at $\mathrm{E}^{*} / \mathrm{A}=3-5 \mathrm{MeV}$ [31].

In Ref. [32] the value of the critical temperature is estimated from the data on the fission probabilities. In this paper the temperature dependence of the liquid-drop fission barrier is calculated as in [1]. The critical temperature $T_{c}$ (at which the surface tension vanishes) is taken as a parameter. It is found that the barrier height is very sensitive to the ratio $T / T_{c}$. Experimental data [33] and calculations are compared for highly excited ${ }^{188} \mathrm{Os}$. It is concluded that $T_{\mathrm{c}}$ is definitely higher than $10 \mathrm{MeV}$. The results a of recent paper [13] are in 

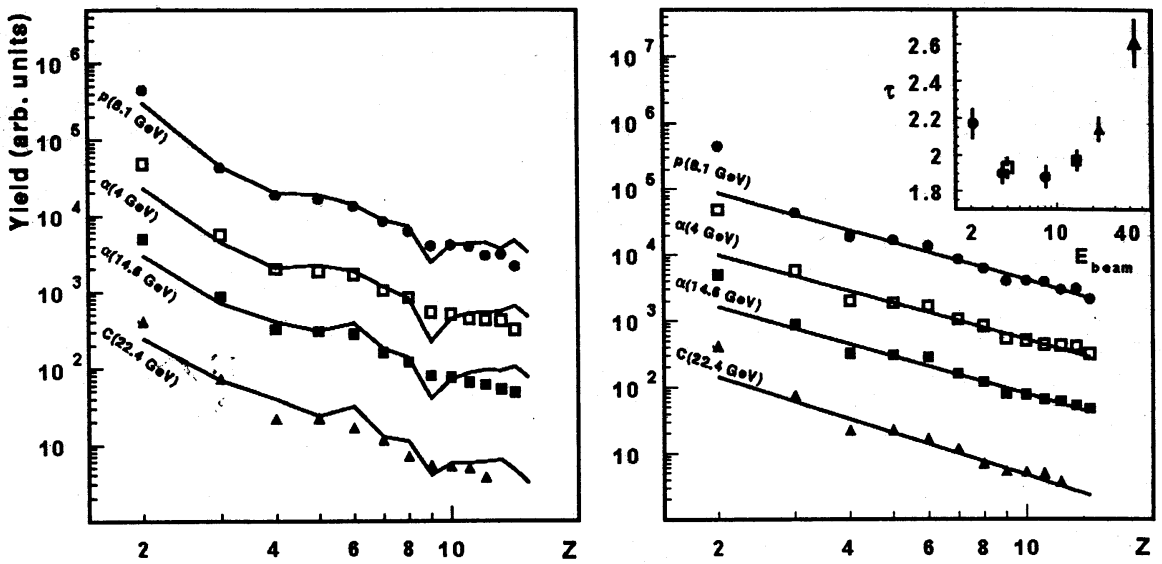

Fig. 8. Fragment charge distributions obtained at $\theta=87^{\circ}$ for $\mathrm{p}+\mathrm{Au}$ at $8.1 \mathrm{GeV}$ (top), ${ }^{4} \mathrm{He}+\mathrm{Au}$ at $4 \mathrm{GeV}$, ${ }^{4} \mathrm{He}+\mathrm{Au}$ at $14.6 \mathrm{GeV}$ and ${ }^{12} \mathrm{C}+\mathrm{Au}$ at $22.4 \mathrm{GeV}$. The lines (left side) are calculated by $\mathrm{NC}+\mathrm{Exp} .+\mathrm{SMM}$ (normalized at $\mathrm{Z}=3$ ). The power law fits are shown on the right panel with $\tau$-parameters given as insert in a function of the beam energy. The last point in insert is for ${ }^{12} \mathrm{C}+\mathrm{Au}$ collisions at $44 \mathrm{GeV}$ (from a preliminary experiment) 
conflict with that conclusion. The fragment mass distributions obtained by the ISIS Collaboration were analyzed in the framework of the Fisher model with the Coulomb energy taken into account. The extracted critical temperature $T_{\mathrm{c}}=6.7 \pm 0.2 \mathrm{MeV}$. We believe that this result should be treated with caution, having in mind the shortcomings of the Fisher model in application to the hot nuclear system [34].

\subsection{Fragment kinetic energy spectra}

The fragment kinetic energy spectra are changing with increasing of the projectile mass. The spectral shapes show an increase in the number of high-energy fragments for heavier projectiles. This observation is summarized in Fig.9 (upper panel), which shows the mean kinetic energies per nucleon as a function of the fragment charge. The figure reveals remarkable enhancement in the kinetic energies for the light fragments $(Z<10)$ emitted in ${ }^{4} \mathrm{He}(14.6 \mathrm{GeV})+\mathrm{Au}$ and ${ }^{12} \mathrm{C}(22.4 \mathrm{GeV})+\mathrm{Au}$ collisions as compared to the $p(8.1 \mathrm{GeV})+\mathrm{Au}$ case. The calculated values (lines) are obtained with the $\mathrm{NC}+$ expansion + SMM combined model. The measured energies are close to the calculated ones for $p+$ Au collisions in the range of fragment charges between 4 and 9 . However, the experimental values for heavier projectiles exceed the theoretical ones, which are similar for all three cases. What is the cause of that?

The kinetic energy of fragments is determined by four terms: thermal motion, Coulomb repulsion, rotation and collective expansion energies, $E=E_{t h}+E_{C}+E_{\text {rot }}+E_{\text {flow. }}$. The Coulomb term is significantly larger than the thermal one as was shown in $[18,23]$. The contribution of the collective rotational energy is negligible even for $\mathrm{C}+\mathrm{Au}$ collisions $[16,26]$. We suggest that the observed energy enhancement is caused by the expansion flow in the system, which is assumed to be radial as the velocity plot (Fig.3) does not show any significant deviation from circular symmetry. Note that the contribution of the collective flow for $p(8.1 \mathrm{GeV})+\mathrm{Au}$ collisions is inconspicuous. As was estimated in [15], the mean flow velocity for that case is less than $0.02 c$. We believe that the observed flow for heavier projectiles is driven by the thermal pressure, which is expected to be larger than for the proton beam. 


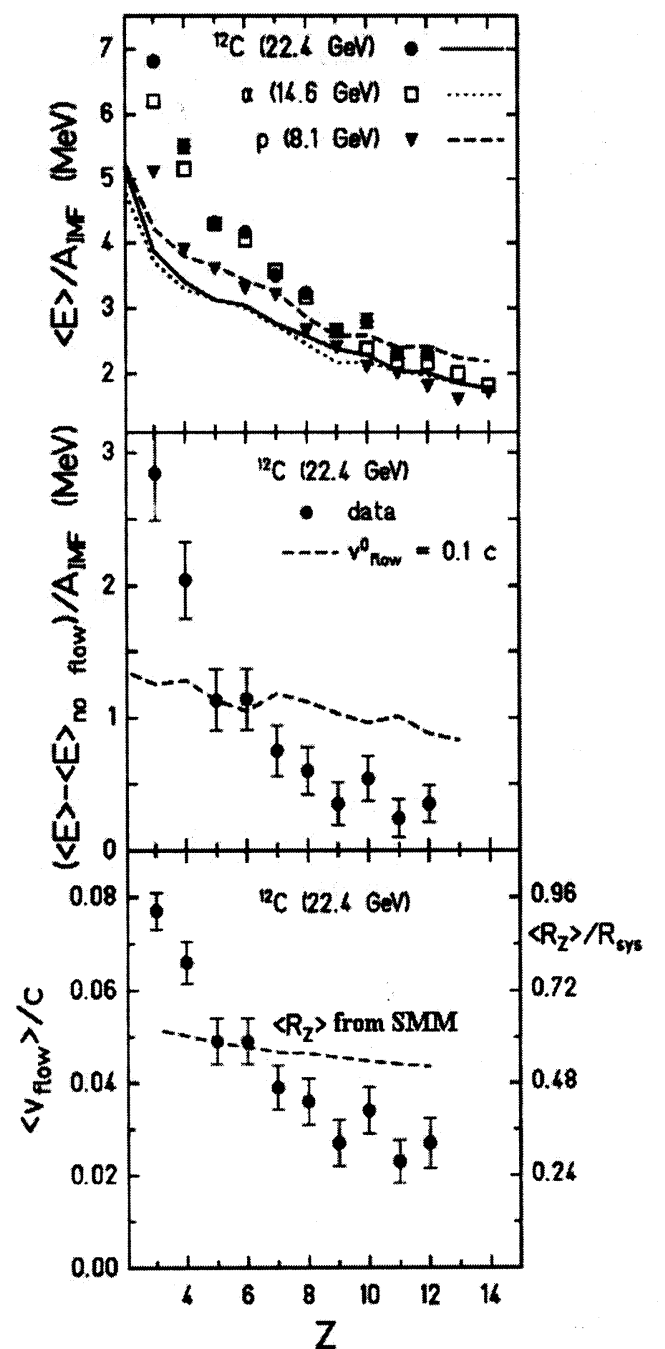

Fig.9. Upper part: Mean kinetic energies of fragments per nucleon measured at $\theta=87^{\circ}$ for $\mathrm{p}(8.1$ $\mathrm{GeV}),{ }^{4} \mathrm{He}(14.6 \mathrm{GeV})$ and ${ }^{12} \mathrm{C}(22.4 \mathrm{GeV})$ collisions with $\mathrm{Au}$. The lines are calculated within INC + Exp. + SMM approach assuming no flow. Middle part: Flow energy per nucleon (dots) obtained as a difference of the measured fragment kinetic energies and the values calculated under assumption of no flow in the system. The dashed line represents a calculation assuming a linear radial profile for the expansion velocity with $v_{\text {flow }}^{0}=0.1 \mathrm{c}$. Lower part: Experimentally deduced mean flow velocities (dots) for ${ }^{12} \mathrm{C}+\mathrm{Au}$ collisions as a function of the fragment charge (left scale), and the mean relative radial coordinates of fragments (right scale), obtained under assumption of a linear radial profile for the expansion velocity. The dashed line shows the mean radial coordinate according to SMM. 
An estimate of the fragment flow energies may be obtained as a difference between the measured IMF energies and those calculated without taking into account any flow in the system. This difference for ${ }^{12} \mathrm{C}+\mathrm{Au}$ collisions is shown in Fig.9 (middle panel).

In an attempt to describe the data we modified the SMM code in the INC + Exp. + SMM concept by including a radial velocity boost for each particle at freeze out. In other words, the radial expansion velocity was superimposed on the thermal motion in the calculation of the multibody Coulomb trajectories. Self-similar radial expansion is assumed, when the local flow velocity is linearly dependent on the distance of the particle from the center of mass. The expansion velocity of particle $Z$ located at radius $\boldsymbol{R}_{Z}$ is given by the following expression:

$$
\boldsymbol{v}_{\text {flow }}(\mathrm{Z})=v_{\text {flow }}^{\mathrm{o}} \cdot \boldsymbol{R}_{Z} / R_{\text {sys }}
$$

where $v^{\circ}$ flow is the radial velocity on the surface of the system. Note that in this case the density distribution is changing in dynamic evolution in a self-similar way being a function of the scaled radius $R_{Z} / R_{\text {sys }}$. The use of the linear profile of the radial velocity is motivated by the hydrodynamic model calculations for an expanding hot nuclear system (see for example Ref.[35]). The value of $v_{\text {flow }}^{o}$ was adjusted to describe the mean kinetic energy measured for the carbon fragment.

Figure10 shows the comparison of the measured and calculated energy spectra (for ${ }^{12} \mathrm{C}+\mathrm{Au}$ collisions) assuming $v_{\text {flow }}^{o}=0.1 c$. The agreement is very good. The calculation without a flow deviates strongly. There is a longstanding problem of a qualitative difference between the chemical or thermal equilibrium temperature and the "kinetic" or so called "slope temperature". A recent discussion of that point can be found in [36]. The mean equilibrium temperature obtained in our calculations is $6.9 \mathrm{MeV}$. At the same time, the slope temperature found from the spectrum shape is $\mathrm{T}_{\mathrm{s}}=14.5 \mathrm{MeV}$ for the "no flow" case (see dashed curve in insert). This is the mutual result of the thermal motion, Coulomb repulsion during the volume disintegration and the secondary decay of the excited fragments. Introducing a rather modest radial flow results in an increase of the slope temperature up to $T_{\mathrm{s}}=24 \mathrm{MeV}$.

Let's return to Fig.9 (middle). The model-calculated flow energy is given as a difference of the calculated fragment energies obtained for $v_{\text {flow }}^{o}=0.1 c$ and $v_{\text {flow }}^{o}=0$. The data deviate significantly from the calculated values for $\mathrm{Li}$ and $\mathrm{Be}$. It may be caused in part by the 


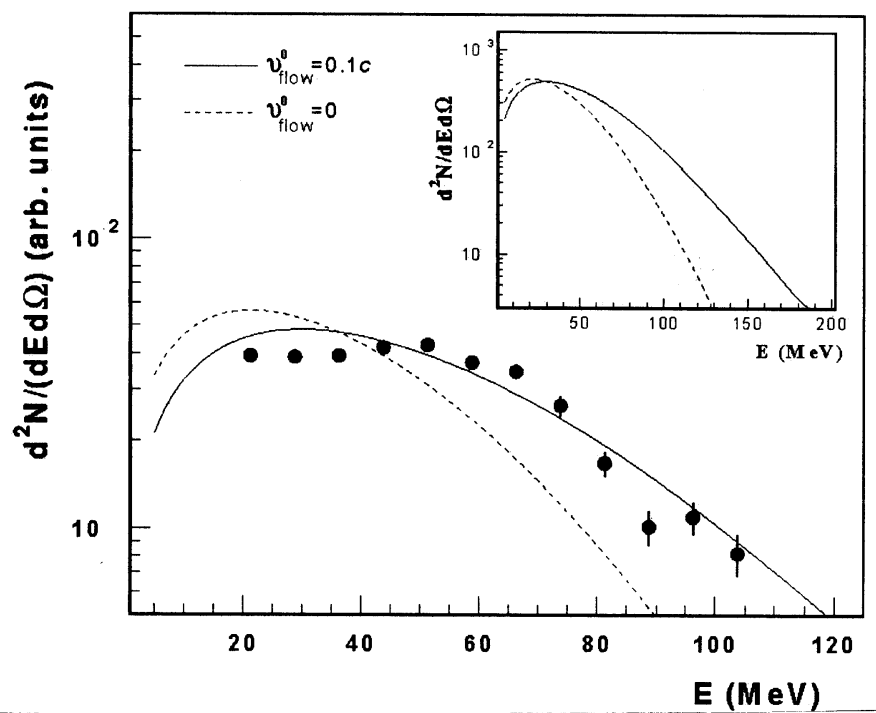

Fig. 10. Energy distribution of carbon fragments $\left(\right.$ at $\theta=87^{\circ}$ ) from ${ }^{12} \mathrm{C}+\mathrm{Au}$ collisions. Solid lines are calculated assuming the radial flow with the velocity on the surface equal to $0.1 c$. Dashed lines are calculated assuming no flow.

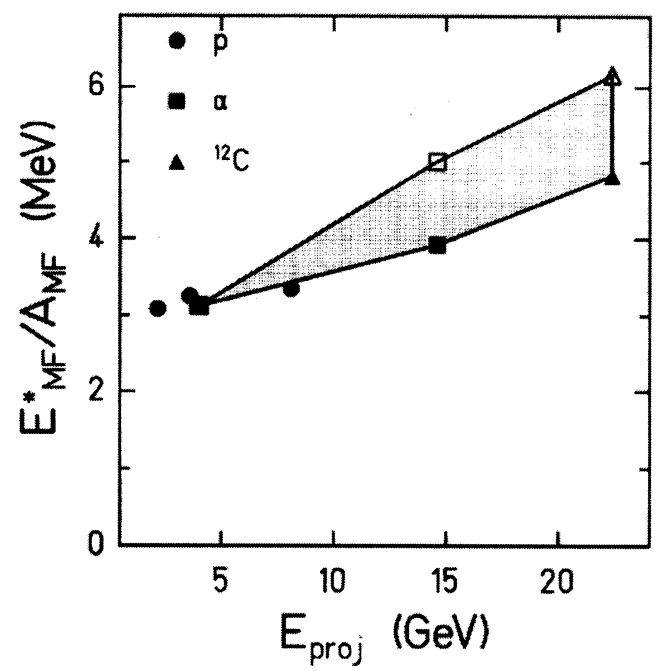

Fig. 11. Mean excitation energy of the fragmenting nucleus per nucleon $E_{M F}^{*} / A_{M F}$ as a function of the beam energy: the solid points refer to the thermal part, the flow energy added is shown as open symbols and grey area. 
contribution of particle emission during the early stage of expansion from a hotter and denser system. This explanation is supported by the fact that the extra energy of $\mathrm{Li}$ fragments with respect to the calculated value is clearly seen in Fig.9 (top) even for the proton-induced fragmentation, where no significant flow is expected.

As to fragments heavier than carbon, the calculated curve in Fig.9 (middle) is above the data and only slightly goes down with increasing fragment charge. This trend of the calculations is to be expected. The mean fragment flow energy is proportional to $\left\langle R Z_{Z}^{2}>\right.$. This value varies only slightly with the fragment charge in the SMM code due to the assumed equal probability for fragments of a given charge to be formed at any point of the available break-up volume. This assumption is a consequence of the model simplification that considers the system as an uniform one with $\rho(r)=$ const for $r \leq R_{\text {sys. }}$. The data in Fig.9 indicate that it is not the case. In fact, the dense interior of the expanded nucleus may favor the appearance of larger IMF's if fragments are formed via the density fluctuations. This observation is also in accordance with the analysis of the mean IMF energies performed in [15] for proton induced fragmentation. It is also seen in Fig.9 (top) that for

$p+$ Au collisions the measured energies are below the theoretical curve for fragments heavier than $\mathrm{Ne}$. This may be explained by the preferential location of the heavier fragments in the interior region of the freeze-out volume, where the Coulomb field is reduced.

The experimentally deduced mean flow velocities of IMF's for ${ }^{12} \mathrm{C}+\mathrm{Au}$ collisions are presented in Fig.9 (lower panel). The values for $\mathrm{Li}$ and $\mathrm{Be}$ are considered as upper limits because of the possible contribution of the preequilibrium emission. The corresponding values of $\left\langle R_{Z} / R_{s y s}\right\rangle$, obtained under the assumption of the linear radial profile for the expansion velocity, can be read on the right-hand scale of the figure. The dashed line shows the mean radial coordinates of the fragments according to the SMM code. The calculated values of $\left\langle R_{Z} / R_{s y s}>\right.$ are only slightly decreasing with $Z$ in contrast to the data.

The total expansion energy can be estimated by integrating the nucleon flow energy over the available volume at freeze out. For a uniform system one gets:

$$
E_{\text {flow }}^{\text {tot }}=(3 / 10) A \cdot m_{N} \cdot\left(v_{\text {flow }}^{o}\right)^{2}\left(1-r_{o} / R_{\text {sys }}\right)^{5}
$$

where $m_{N}$ and $r_{o}$ are the nucleon mass and radius. For ${ }^{12} \mathrm{C}+\mathrm{Au}$ collisions it gives $E^{\text {tot }}{ }_{\text {flow }} \cong$ $115 \mathrm{MeV}$, corresponding to the flow velocity on the surface equal to $0.1 c$. 
Similar results are obtained for ${ }^{4} \mathrm{He}(14.6 \mathrm{GeV})+\mathrm{Au}$ collisions. The excitation energies of the fragmenting systems studied are largely thermal ones, therefore we deal with the thermal multifragmentation. It is reflected in Fig. 11 where the mean total excitation energy per nucleon $E_{M F} / A_{M F}$ is shown as a function of the incident energy.

The full symbols correspond to the thermal part of the excitation energy obtained via analysis of the data on fragment multiplicity and charge distributions with the combined model of the process. Open symbols include the flow energy. Thermal energies for these cases are four times larger than collective ones. The onset of the collective flow driven by the thermal pressure takes place at the excitation energy around $4 \mathrm{MeV} /$ nucleon, which is in good agreement with the results of [37]. The mean fragmenting masses are equal to 158 , 103,86 for proton $(8.1 \mathrm{GeV}),{ }^{4} \mathrm{He}(14.6 \mathrm{GeV})$ and ${ }^{12} \mathrm{C}(22.4 \mathrm{GeV})$ collisions with $\mathrm{Au}$ respectively. Note that selection of the events with the IMF multiplicity $M \geq 2$ (for the correlation measurements) results in an increase in the mean excitation energy by $0.5-0.7$ $\mathrm{MeV} /$ nucleon [23]:

\section{Conclusion}

In this work we study the mechanism of multifragment emission in collisions of relativistic protons, ${ }^{4} \mathrm{He}$ and ${ }^{12} \mathrm{C}$ with an Au target. The data obtained support the interpretation of this phenomenon as "thermal multifragmentation", which is a statistical break-up process of a diluted and hot system with the density 3-4 times smaller than the normal one. Thermal multifragmentation is a new multibody decay mode of an extremely excited nucleus with a very short life time. It was found via IMF-IMF relative angle correlations that the fragment mean emission time $\tau_{e m} \leq 70 \mathrm{fm} / c$. This decay process can be interpreated as the first order nuclear "liquid-fog" phase transition inside the spinodal region ( spinodal decomposition).

The evolution of the thermal multifragmentation mechanism with increasing projectile mass was investigated. The onset of radial collective flow was observed for heavier projectiles. It is believed to be driven by the thermal pressure. The mean total flow energy at the break-up moment is estimated to be around $115 \mathrm{MeV}$ for both ${ }^{4} \mathrm{He}(14.6 \mathrm{GeV})$ and ${ }^{12} \mathrm{C}$ $(22.4 \mathrm{GeV})$ beams, while the mean thermal excitation is around $400 \mathrm{MeV}$. 
The flow energy of fragments decreases as their charge increases. The analysis of the data reveals interesting information on the fragment space distribution inside the break-up volume: heavier IMF are formed predominately in the interior of the fragmenting nucleus possibly due to the density gradient. This conclusion is in contrast to the predictions of the Statistical Multifragmentation Model.

This study of multifragmentation using a range of projectiles demonstrates a transition from pure "thermal decay" (for $p+$ Au collisions) to disintegration "decorated" by the onset of a collective flow (for heavier projectiles ). Nevertheless, the decay mechanism should be considered as thermal multifragmentation. The partition of the system is governed by the nuclear heating, and IMF charge distributions in all the cases considered are well described by the statistical model neglecting any flow.

The authors are thankful to Profs. A. Hrynkiewicz, A.N. Sissakian, S.T. Belyaev, A.I. Malakhov, and N.A. Russakovich for support and to the staff of the JINR synchrophasotron for running the accelerator. The research was supported in part by Grant No 00-02-16608 from the Russian Foundation for Basic Research, by the Grant of the Polish Plenipotentiary in JINR, by Grant NATO PST.CLG.976861, by Grant No1P03 12615 from the Polish State Committee for Scientific Research, by Contract No 06DA453 with Bundesministerium fuer Forschung und Technologie and by the US National Science Foundation.

\section{References}

1. G. Sauer, H. Chandra and U. Mosel, Nucl. Phys., A264, 221 (1976).

2. H. Jaqaman, A.Z. Mekjian, L. Zamick, Phys. Rev., C27, 2782 (1983).

3. P.J. Siemens, Nature, 305, 410 (1983); Nucl. Phys., A428, 189c (1984).

4. A. Guarnera, B. Jacquot, Ph. Chomaz, M. Colonna, XXXIII Winter Meeting on Nucl. Phys., Bormio, 1995; Preprint GANIL P 95-05 (Caen,1995).

5. S.J. Lee and A.Z. Mekjian, Phys. Rev., C56, 2621 (1997).

6. V. Baran, M. Colonna, M. Di Toro, A.B. Larionov, Nucl. Phys., A632, 287 (1998).

7. M. D'Agostino et al.,Nucl.Phys., A650, 329 (1999); Phys Lett., B473, 219 (2000).

8. L. Beaulieu et al., Phys. Rev. Lett., 84, 5971 (2000).

9. O. Lopez, Nucl. Phys., A685, 246c (2001). 
10. B. Borderie et al., Phys. Rev. Lett., 863252 (2001).

11. A. Bonasera, M. Bruno, C.O. Dorso and P.F. Mastinu, La Rivista del Nuovo Chimento, 23, №2, 1 (2000).

12. J. Richert, P. Wagner, Phys. Reports, 350, № 1, 1 (2001).

13. J.B. Elliott, L.G. Moretto, L. Phair et al., Preprint LBNL-47711 (Berkeley, 2001).

14. S.P. Avdeyev et al., Nucl. Instrum. Meth., A332, 149 (1993).

S.P. Avdeyev et al., Pribory i Tekhnika Eksper., 39, 7 (1996); (Instr. Exp. Techn., 39, 153 (1996)).

15. S.P. Avdeyev et al., Eur. Phys. J., A3, 75 (1998).

16. S.P. Avdeyev et al., Yad. Fiz., 64, 1628 (2001) (Phys. of Atom. Nuclei, 64, 1549 (2001)).

17. V. Lips et al.,,Phys. Rev. Lett., 72, 1604 (1994).

18. V. Lips et al., Phys. Lett., B338, 141 (1994).

S.Y. Shmakov et al., Yad. Fiz., 58, 1735 (1995) (Phys. of Atom. Nucl., 58, 1635 (1995)).

19. Bao-An Li et al., Phys. Lett., B335, 1 (1994).

20. V.D. Toneev, et al., Nucl. Phys., A519, 463c (1990).

N.S. Amelin et al., Yad. Fiz., 52, 272 (1990).

21. J. Bondorf et al., Phys. Rev., 257, 133 (1995); Nucl. Phys., A444, 476 (1985).

22. O. Shapiro, D.H.E. Gross, Nucl. Phys., A573, 143 (1994).

23. V.K. Rodionov et al., Nucl. Phys., A700, 457 (2002).

24. W.A. Friedman, Phys. Rev., C42, 667 (1990).

25. W. Reisdorf, Progr. Theo. Phys. Suppl., No. 140, 111 (2000).

26. S.P. Avdeyev et al., Phys. Lett., B503, 256 (2001).

27. A.S. Hirsh et al., Phys.Rev., C29, 508 (1984).

28. A.D. Panagiotou, M.W. Curtin and D.K. Scott, Phys.Rev., C31, 55 (1985).

29. N.T. Porile et al., Phys Rev., C39, 1914 (1989).

30. M.E. Fisher, Physics, 3, 255 (1967).

31. V.A. Karnaukhov et al.,Yad. Fiz., 62, 272 (1999) (Phys. of At. Nuclei, 62, 237 (1999)).

32. V.A. Karnaukhov, Yad. Fiz., 60, 1780 (1997) (Phys. of At. Nuclei, 60, 1625 (1997)).

33. L.G. Moretto et al., Phys. Letter, B38, 471 (1972). 
34. J. Schmelzer, G. Roepke, F.- P. Ludwig, Phys. Rev., C55, 1917 (1997).

35. J.P. Bondorf et al., Nucl. Phys., A296, 320 (1978).

36. T. Odeh et al., Phys. Rev. Lett., 84, 4557 (2000).

37. T. Lefort et al., Phys. Rev., C62, 031604 (2000).

Received on April 11, 2002. 
Карнаухов В. А. и др.

Тепловая мультифрагментация горячих ядер

и фазовый переход «жидкость-туман»

С помощью $4 \pi$-установки ФАЗА исследовалась множественная эмиссия фрагментов с промежуточной массой (ФПМ) в соударениях протонов (до 8,1 ГэВ), ${ }^{4} \mathrm{He}$ (4 и 14,6 ГэВ) и ${ }^{12} \mathrm{C}(22,4$ ГэВ) с мишенью из золота. Во всех случаях имеет место тепловая мультифрагментация горячего спектатора мишени. Распределение ФПМ по множественности и заряду хорошо описывается комбинированной моделью, включающей внутриядерный каскад с последующим статистическим многотельным распадом возбужденного остаточного ядра. Этот процесс происходит за время $\tau \leq 70$ фм/с, что установлено путем анализа угловых ФПМ-ФПМ-корреляций. Множественная эмиссия фрагментов интерпретируется как фазовый переход «жидкость-туман», происходящий в спинодальной области.

Исследована эволюция механизма тепловой мультифрагментации с ростом массы бомбардируюшей частицы. Отмечено появление радиального коллективного потока для пучков ${ }^{4} \mathrm{He} \mathrm{и}{ }^{12} \mathrm{C}$. Из результатов анализа следует, что более тяжелые фрагменты образуются преимущественно в центральной области распадного объема.

Работа выполнена в Лаборатории ядерных проблем им. В. П. Джелепова ОИЯИ.

Препринт Объединенного института ядерных исследований. Дубна, 2002

Karnaukhov V. A. et al.

E6-2002-75

Thermal Multifragmentation of Hot Nuclei and Liquid-Fog Phase Transition

Multiple emission of intermediate-mass fragments in the collisions of protons (up to $8.1 \mathrm{GeV}),{ }^{4} \mathrm{He}(4$ and $14.6 \mathrm{GeV})$ and ${ }^{12} \mathrm{C}(22.4 \mathrm{GeV})$ on $\mathrm{Au}$ has been studied with the $4 \pi$-setup FASA. In all cases thermal multifragmentation of the hot and diluted target spectator takes place. The fragment multiplicity and charge distributions are well described by the combined model including the modified intranuclear cascade followed by the statistical multibody decay of the hot system. IMF-IMF correlation study supports this picture giving very short time scale of the process $(\tau \leq 70 \mathrm{fm} / \mathrm{c})$. This decay process can be interpreted as the first order nuclear liquid-fog phase transition inside the spinodal region.

The evolution of the mechanism of thermal multifragmentation with increasing projectile mass was investigated. The onset of the radial collective flow was observed for heavier projectiles. The analysis reveals the information on the fragment space distribution inside the break-up volume: heavier IMF are formed predominantly in the interior of the fragmenting nucleus possibly due to the density gradient.

The investigation has been performed at the Dzhelepov Laboratory of Nuclear Problems, JINR. 
Макет T. E. Попеко

ЛР № 020579 от 23.06.97.

Подписано в печать 30.05.2002.

Формат $60 \times 90 / 16$. Бумага офсетная. Печать офсетная.

Усл. печ. л. 1,68. Уч.-изд. л. 2,3. Тираж 320 экз. Заказ № 53311.

Издательский отдел Объединенного института ядерных исследований

141980, г. Дубна, Московская обл., ул. Жолио-Кюри, 6. 